Annales Academiæ Scientiarum Fennicæ

Mathematica

Volumen 39, 2014, 941-946

\title{
A MARTENS-TYPE THEOREM IN THE CONTEXT OF COHOMOLOGY OF RIEMANN SURFACES
}

\author{
Masaharu Tanabe \\ Tokyo Institute of Technology, Department of Mathematics \\ Ohokayama, Meguro, Tokyo, 152-8551, Japan; tanabe.m.ac@m.titech.ac.jp
}

\begin{abstract}
A holomorphic map between closed Riemann surfaces is completely determined by the induced homomorphism of the first homology groups with integer coefficients. This is a consequence of Martens' theorem proven in [6]. In this paper, We study holomorphic maps of closed Riemann surfaces in the context of cohomology and extend Martens' theorem.
\end{abstract}

\section{Introduction}

A classic theorem of Hurwitz [4] states that if an automorphism of a closed Riemann surface of genus greater than one induces the identity on the first homology group then the automorphism is the identity. Accola [1] and Gilman [3] generalized this theorem.

They studied automorphisms of Riemann surfaces and proved several theorems concerning rigidity of automorphisms in terms of homology groups. One of their results interesting is the following which firstly proved by Accola and later Gilman proved a theorem which includes it as a corollary. In the following theorem, $\chi_{i} \cdot \chi_{j}$ denotes the intersection number of $\chi_{i}$ and $\chi_{j}$.

Theorem 1.1. (Accola) Let $X$ be a closed Riemann surface of genus greater than one. Let $T$ be an automorphism of $X$. Suppose that there exist four independent cycles $\chi_{1}, \chi_{2}, \chi_{3}, \chi_{4}$ so that $\chi_{1} \cdot \chi_{3}=1, \chi_{2} \cdot \chi_{4}=1$, otherwise $\chi_{i} \cdot \chi_{j}=0$ and that $T\left(\chi_{i}\right)=\chi_{i}$ for $i=1,2,3,4$. Then $T$ is the identity.

Martens [7] proposed several problems in the theory of closed Riemann surfaces and one topic was about their results. He wrote that it would be interesting to try and interpret their results in the context of Jacobian varieties.

Martens [6] himself generalized Hurwitz' theorem for holomorphic maps between closed Riemann surfaces of possibly different genera.

Theorem 1.2. (Martens) Let $\tilde{X}, X_{1}, X_{2}$ be closed Riemann surfaces of genera $\geq 1$ and let $f_{i}: \widetilde{X} \rightarrow X_{i}(i=1,2)$ be non-constant holomorphic maps. Assume that there exists a homomorphism $H$ of the first homology groups from $H_{1}\left(X_{1}, \mathbf{Z}\right)$ onto $H_{1}\left(X_{2}, \mathbf{Z}\right)$ which commutes with the induced homomorphisms $f_{i *}: H_{1}(\widetilde{X}, \mathbf{Z}) \rightarrow$ $H_{1}\left(X_{i}, \mathbf{Z}\right)(i=1,2)$, i.e., $f_{2 *}=H \circ f_{1 *}$. Then there exists a unique (modulo a translation in genus 1) holomorphic map $h: X_{1} \rightarrow X_{2}$ which induces the given homomorphism $H$, and $h$ satisfies $f_{2}=h \circ f_{1}$.

Interpreting Theorem 1.1 and Theorem 1.2 in terms of $H^{1}(X, \mathbf{Z})$ (the dual for $H_{1}(X, \mathbf{Z})$, that is to say in the context of dual Jacobian varieties rather than Jacobian varieties), we can generalize these theorems for holomorphic maps of closed Riemann

doi:10.5186/aasfm.2014.3938

2010 Mathematics Subject Classification: Primary 14F40, 14H40, 30 F30.

Key words: Riemann surfaces, cohomology, Jacobian varieties. 
surfaces [10].

Theorem 1.3. Let $\widetilde{X}, X_{1}, X_{2}$ be closed Riemann surfaces of genera greater than one and let $f_{i}: \widetilde{X} \rightarrow X_{i}(i=1,2)$ be non-constant holomorphic maps. Assume that there exist $a_{i}, b_{i} \in H^{1}\left(X_{i}, \mathbf{Z}\right)(i=1,2)$ so that $\iint_{X_{i}} a_{i} \wedge b_{i}=1(i=1,2)$ and that $f_{1}^{*} a_{1}=f_{2}^{*} a_{2}$ and $f_{1}^{*} b_{1}=f_{2}^{*} b_{2}$ in $H^{1}(\tilde{X}, \mathbf{Z})$. Then there exists a conformal map $h: X_{1} \rightarrow X_{2}$ which satisfies $f_{2}=h \circ f_{1}$.

The purpose of this paper is to present the following Martens-type theorem which is in the context of cohomology.

Theorem 1.4. Let $\tilde{X}, X_{1}, X_{2}$ be closed Riemann surfaces of genera greater than one and let $f_{i}: \widetilde{X} \rightarrow X_{i}(i=1,2)$ be non-constant holomorphic maps and $f_{i}^{*}: H^{1}\left(X_{i}, \mathbf{C}\right) \rightarrow H^{1}(\widetilde{X}, \mathbf{C})$ be the induced homomorphisms of the cohomology groups. Assume that there exists a homomorphism $\tau: H^{1}\left(X_{2}, \mathbf{C}\right) \rightarrow H^{1}\left(X_{1}, \mathbf{C}\right)$ such that $f_{2}^{*}=f_{1}^{*} \circ \tau$ holds. Then there exists a unique holomorphic map $h: X_{1} \rightarrow X_{2}$ such that $h^{*}=\tau$, and $h$ satisfies $f_{2}=h \circ f_{1}$.

From Theorem 1.4, one can derive the following without much effort.

Corollary 1.5. Let $\widetilde{X}, X_{1}, X_{2}$ be closed Riemann surfaces of genera greater than one and let $f_{i}: \widetilde{X} \rightarrow X_{i}(i=1,2)$ be non-constant holomorphic maps and $f_{i}^{*}: \mathcal{H}^{1}\left(X_{i}\right) \rightarrow \mathcal{H}^{1}(\widetilde{X})$ be the induced homomorphisms of the spaces of holomorphic differential 1-forms. Assume that there exists a homomorphism $\tau: \mathcal{H}^{1}\left(X_{2}\right) \rightarrow \mathcal{H}^{1}\left(X_{1}\right)$ such that $f_{2}^{*}=f_{1}^{*} \circ \tau$ holds. Then there exists a unique holomorphic map $h: X_{1} \rightarrow X_{2}$ such that $h^{*}=\tau$, and $h$ satisfies $f_{2}=h \circ f_{1}$.

As an application of Theorem 1.4, we have the following which is an extension of Theorem 1.2. We assume here that the genera of Riemann surfaces are greater than one, although Theorem 1.2 does not exclude the case where genus is one.

Corollary 1.6. Let $\widetilde{X}, X_{1}, X_{2}$ be closed Riemann surfaces of genera greater than one and let $f_{i}: \widetilde{X} \rightarrow X_{i}(i=1,2)$ be non-constant holomorphic maps. Assume that there exists a homomorphism of the first homology groups with Q-coefficients $H: H_{1}\left(X_{1}, \mathbf{Q}\right) \rightarrow H_{1}\left(X_{2}, \mathbf{Q}\right)$ which commutes with the induced homomorphisms $f_{i *}: H_{1}(\widetilde{X}, \mathbf{Q}) \rightarrow H_{1}\left(X_{i}, \mathbf{Q}\right)(i=1,2)$, i.e., $f_{2 *}=H \circ f_{1 *}$. Then there exists a unique holomorphic map $h: X_{1} \rightarrow X_{2}$ which induces the given homomorphism $H$, and $h$ satisfies $f_{2}=h \circ f_{1}$.

In [6] Martens made mention of the following problem: Given a holomorphic map of closed Riemann surfaces $f: \widetilde{X} \rightarrow X$ and an automorphism $T$ of $\widetilde{X}$, find a condition for the existence of an automorphism $\tau$ of $X$ with $\tau \circ f=f \circ T$.

If the induced homomorphism

$$
f_{*}: H_{1}(\widetilde{X}, \mathbf{Z}) \rightarrow H_{1}(X, \mathbf{Z})
$$

is represented by a matrix $N$ and

$$
T_{*}: H_{1}(\tilde{X}, \mathbf{Z}) \rightarrow H_{1}(\tilde{X}, \mathbf{Z})
$$

is represented by a matrix $M$, the existence of such $\tau$ is equivalent to the existence of an integer matrix $M^{\prime}$ such that $M^{\prime} N=N M$ by Theorem 1.2. But by Corollary 1.6, the problem is just to find a solution of $M^{\prime} N=N M$ in Q-coefficient matrices so now it is a problem of linear algebra. 
Another application of Theorem 1.4 is about divisors of meromorphic functions.

Corollary 1.7. Let $X$ be a closed Riemann surface of genus greater than one and let $n$ be an integer greater than one. There are at most finitely many pairs of points $(p, q) \in X \times X$ such that $n q-n p$ is a principle divisor, i.e., there exists a meromorphic function $f$ on $X$ with $(f)=n q-n p$.

From the Weierstrass gap Theorem, if we drop the assumption of the zero divisor, for an arbitrary $p \in X$ and an arbitrary $n \geq 2 g$ where $g$ is the genus of $X$, there exits a meromorphic function whose polar divisor is $n p$.

The proof of Theorem 1.4 uses Jacobian varieties and the canonical map from a closed Riemann surface to a projective space. First, we will obtain a relation between homomorphisms of Jacobian varieties under the theorem's assumption. Then we will observe their actions on embedded Riemann surfaces in Jacobians to compare with the canonical map from a Riemann surface to a projective space.

\section{Preliminaries}

First, we recall some notions from complex tori (for details, see e.g. [5]). Let $V$ be a complex vector space of dimension $n$ and $\Gamma$ a lattice in $V$. The quotient $T=V / \Gamma$ is called a complex torus of dimension $n$. Denote by $\widehat{T}=V^{*} / \widehat{\Gamma}$ the dual where $V^{*}$ is the space of $\mathbf{C}$-antilinear functionals on $V$ and $\widehat{\Gamma}=\left\{l \in V^{*}: \operatorname{Im} l(\Gamma) \subseteq \mathbf{Z}\right\}$ is the dual lattice of $\Gamma$. We have

$$
\widehat{\widehat{T}}=T \text {. }
$$

Let $\mathfrak{f}$ be a homomorphism between two complex tori $T=V / \Gamma$ and $T^{\prime}=V^{\prime} / \Gamma^{\prime}$. Then, there is a unique C-linear map $F: V \rightarrow V^{\prime}$ with $F(\Gamma) \subseteq \Gamma^{\prime}$ inducing $\mathfrak{f}$. We call $F$ the analytic representation of $\mathfrak{f}$. For the analytic representation $F: V \rightarrow V^{\prime}$ of a homomorphism $\mathfrak{f}: T \rightarrow T^{\prime}$, the dual map ${ }^{t} F: V^{\prime *} \rightarrow V^{*}$ associating to an antilinear functional $l \in V^{\prime *}$ the antilinear functional $l \circ F \in V^{*}$ induces a homomorphism $\widehat{\mathfrak{f}}: \widehat{T}^{\prime} \rightarrow \widehat{T}$, since ${ }^{t} F\left(\widehat{\Gamma}^{\prime}\right) \subseteq \widehat{\Gamma}$. We call $\widehat{\mathfrak{f}}$ the dual map of $\mathfrak{f}$. We note

$$
\widehat{\widehat{\mathfrak{f}}}=\mathfrak{f} \text {. }
$$

If $\mathfrak{h}: T^{\prime} \rightarrow T^{\prime \prime}$ is another homomorphism of complex tori, then

$$
\widehat{\mathfrak{h} \mathfrak{f}}=\widehat{\mathfrak{f}} \widehat{\mathfrak{h}} \text {. }
$$

For any positive integer $n$ define an endomorphism of a complex torus

$$
n_{T}: T \rightarrow T
$$

by

$$
x \mapsto n x .
$$

Then

$$
\widehat{n_{T}}=n_{\widehat{T}} .
$$

Now we turn to Riemann surfaces. General references for the theory of Riemann surfaces are Farkas and Kra [2], Springer [9], and Miranda [8] for the language of algebraic geometry. Let $X$ be a closed Riemann surface of genus $g>1$. Denote by $\mathcal{H}^{1}(X)$ the space of holomorphic differential 1-forms on $X$.

$$
H^{1}(X, \mathbf{Z}) \hookrightarrow H^{1}(X, \mathbf{C}) \cong \mathcal{H}^{1}(X) \oplus \overline{\mathcal{H}^{1}(X)}
$$

holds. 
Set $\Omega=\operatorname{Hom}\left(\mathcal{H}^{1}(X), \mathbf{C}\right)$. The Jacobian variety $J(X):=\Omega / H_{1}(X, \mathbf{Z})$ is a complex torus of dimension $g$, and considering $\overline{\mathcal{H}}:=\overline{\mathcal{H}^{1}(X)}$ of $\mathbf{C}$-antilinear forms on $\Omega$, we will denote by $\widehat{J(X)}=\overline{\mathcal{H}} / H^{1}(X, \mathbf{Z})$ the dual Jacobian variety.

Let $\left\{\zeta_{1}, \cdots, \zeta_{g}\right\}$ be a basis for $\mathcal{H}^{1}(X)$. Identify $\Omega=\mathbf{C}^{g}$. Then the Abel-Jacobi map

is given by

$$
\varphi_{X}: X \rightarrow \mathbf{C}^{g} / H_{1}(X, \mathbf{Z})
$$

$$
\varphi_{X}(p)={ }^{t}\left(\int_{p_{0}}^{p} \zeta_{1}, \cdots, \int_{p_{0}}^{p} \zeta_{g}\right)
$$

where $p_{0} \in X$ is fixed. This map is an embedding. For any given holomorphic map between closed Riemann surfaces

$$
f: \widetilde{X} \rightarrow X
$$

there exists a homomorphism between Jacobians

$$
\mathfrak{f}: J(\tilde{X}) \rightarrow J(X)
$$

with

The canonical map for $X$

$$
\mathfrak{f} \circ \varphi_{\widetilde{X}}=\varphi_{X} \circ f
$$

$$
\phi_{X}: X \rightarrow \mathbf{P}^{g-1}
$$

is defined by $\phi_{X}(P)=\left(\zeta_{1}(P), \cdots, \zeta_{g}(P)\right)$ in homogeneous coordinates. The following is well known (see e.g. [2], [8]).

Theorem 2.1. The canonical map is an embedding if and only if $X$ is not hyperelliptic. For a hyperelliptic $X$, the canonical map is a composition of the double covering map $X \rightarrow \mathbf{P}^{1}$ and an embedding $\mathbf{P}^{1} \rightarrow \mathbf{P}^{g-1}$.

\section{Proofs of Theorem 1.4 and its corollaries}

Now we will prove Theorem 1.4. The given homomorphism $\tau$ maps $\mathcal{H}^{1}\left(X_{2}\right)$ to $\mathcal{H}^{1}\left(X_{1}\right)$. Indeed, for an arbitrary $\alpha \in \mathcal{H}^{1}\left(X_{2}\right)$ we can write

$$
\tau \alpha=\beta_{1}+\overline{\beta_{2}}, \quad \beta_{1}, \beta_{2} \in \mathcal{H}^{1}\left(X_{1}\right)
$$

by the decomposition (1), so

$$
f_{1}^{*} \circ \tau(\alpha)=f_{1}^{*}\left(\beta_{1}+\overline{\beta_{2}}\right)=f_{1}^{*} \beta_{1}+f_{1}^{*} \overline{\beta_{2}}=f_{2}^{*} \alpha .
$$

The homomorphisms $f_{1}^{*}, f_{2}^{*}$ are compatible with the decomposition (1) since $f_{1}$ and $f_{2}$ are holomorphic maps. Thus we have $f_{1}^{*} \overline{\beta_{2}}=0$ which is equivalent to $\overline{\beta_{2}}=0$.

Similarly, $\tau$ maps $\overline{\mathcal{H}^{1}\left(X_{2}\right)}$ to $\overline{\mathcal{H}^{1}\left(X_{1}\right)}$.

Let $\alpha \in H^{1}\left(X_{2}, \mathbf{Z}\right)$. Then $\tau \alpha$ must be in $H^{1}\left(X_{1}, \mathbf{Q}\right)$ since $f_{1}^{*} \circ \tau(\alpha)=f_{2}^{*} \alpha \in$ $H^{1}(\tilde{X}, \mathbf{Z})$. Thus there exists a positive integer $n$ such that $n \cdot \tau \alpha \in H^{1}\left(X_{1}, \mathbf{Z}\right)$ for any $\alpha \in H^{1}\left(X_{2}, \mathbf{Z}\right)$ since the cohomology groups are of finite rank. This implies that $n \cdot \tau$ defines a homomorphism of the dual Jacobian varieties and we denote it by

$$
n \tau: \widehat{J\left(X_{2}\right)} \rightarrow \widehat{J\left(X_{1}\right)} \text {. }
$$

We denote by $\mathfrak{f}_{\mathfrak{i}}$ the homomorphisms of Jacobians induced from $f_{i}(i=1,2)$. Then we have a relation between homomorphisms of the dual Jacobians

$$
\widehat{\mathfrak{f}_{2}} \circ n_{\widehat{J\left(X_{2}\right)}}=\widehat{\mathfrak{f}_{1}} \circ n \tau \text {, }
$$


and considering the dual of these maps, we have a relation between homomorphisms of Jacobians

$$
n_{J\left(X_{2}\right)} \circ \mathfrak{f}_{2}=\widehat{n \tau} \circ \mathfrak{f}_{1} .
$$

Let $\left\{\zeta_{1}, \cdots, \zeta_{\gamma_{2}}\right\}$ be a basis for $\mathcal{H}^{1}\left(X_{2}\right)$. For any given local coordinate $z$ around $p \in X_{2}$, we express the differential as

$$
\zeta_{j}=\omega_{j}(z) d z
$$

We put

The restricted map

$$
X^{\prime}=n_{J\left(X_{2}\right)}\left(\varphi_{X_{2}}\left(X_{2}\right)\right)
$$

$$
\left.n_{J\left(X_{2}\right)}\right|_{\varphi_{X_{2}}\left(X_{2}\right)}: \varphi_{X_{2}}\left(X_{2}\right) \rightarrow X^{\prime}
$$

is unramified. Thus we can view $z$ as a local coordinate around

$$
p^{\prime}:=n_{J\left(X_{2}\right)}\left(\varphi_{X_{2}}(p)\right) \in X^{\prime}
$$

(although $X^{\prime}$ may have singularities as a subvariety of $J\left(X_{2}\right)$ ). Then $p^{\prime}$ can be written as

$$
p^{\prime}={ }^{t}\left(n \int_{p_{0}}^{p} \zeta_{1}, \cdots, n \int_{p_{0}}^{p} \zeta_{\gamma_{2}}\right) .
$$

We express $\left.n_{J\left(X_{2}\right)}\right|_{\varphi_{X_{2}}\left(X_{2}\right)}$ in terms of the local coordinate $z$ and differentiate this with respect to $z$ to define

$$
\pi: X^{\prime} \rightarrow \mathbf{P}^{\gamma_{2}-1}
$$

by

$$
\pi\left(p^{\prime}\right)={ }^{t}\left(n \zeta_{1}(p), \cdots, n \zeta_{\gamma_{2}}(p)\right)
$$

in homogenous coordinates and this is expressed as

$$
\pi(z)={ }^{t}\left(n \omega_{1}(z), \cdots, n \omega_{\gamma_{2}}(z)\right)
$$

in terms of the local coordinate. (Strictly speaking, $\pi$ is defined away from singularities if they exist.) From Theorem 2.1, $\pi\left(X^{\prime}\right) \simeq X_{2}$ or $\pi\left(X^{\prime}\right) \simeq \mathbf{P}^{1}$.

If $\pi\left(X^{\prime}\right) \simeq X_{2}$, then $X^{\prime} \simeq X_{2}$. By $(2)$,

$$
n_{J\left(X_{2}\right)} \circ \mathfrak{f}_{2} \circ \varphi_{\widetilde{X}}=\widehat{n \tau} \circ \mathfrak{f}_{1} \circ \varphi_{\widetilde{X}} .
$$

Thus we have

$$
n_{J\left(X_{2}\right)} \circ \varphi_{X_{2}} \circ f_{2}=\widehat{n \tau} \circ \varphi_{X_{1}} \circ f_{1}: \tilde{X} \rightarrow X^{\prime} \simeq X_{2},
$$

and we see that there exists a holomorphic map $h: X_{1} \rightarrow X_{2}$ which satisfies $f_{2}=$ $h \circ f_{1}$. Observing the construction of $h$, we see that the homomorphism $\mathfrak{h}: J\left(X_{1}\right) \rightarrow$ $J\left(X_{2}\right)$ with $\varphi_{X_{2}} \circ h=\mathfrak{h} \circ \varphi_{X_{1}}$ satisfies $\mathfrak{h}(x)=n^{-1} \cdot \widehat{n \tau}(x)$ for all $x \in J\left(X_{1}\right)$. Thus the analytic representation of $\widehat{\mathfrak{h}}$ must be $\left.\tau\right|_{\overline{\mathcal{H}^{1}\left(X_{2}\right)}}$ and this implies that $h^{*}=\tau$. We will show the uniqueness of $h$ such that $h^{*}=\tau$. Suppose that another holomorphic map $h_{1}: X_{1} \rightarrow X_{2}$ satisfies $h_{1}^{*}=\tau$. Then the homomorphism $\mathfrak{h}_{1}: J\left(X_{1}\right) \rightarrow J\left(X_{2}\right)$ induced from $h_{1}$ satisfies $\widehat{\mathfrak{h}_{1}}=\left.\tau\right|_{\overline{\mathcal{H}^{1}\left(X_{2}\right)}}$, hence $\widehat{\mathfrak{h}_{1}}=\widehat{\mathfrak{h}}$. Thus $\mathfrak{h}_{1}=\mathfrak{h}$ and by $\varphi_{X_{2}} \circ h=$ $\mathfrak{h} \circ \varphi_{X_{1}}=\varphi_{X_{2}} \circ h_{1}$, we see that $h_{1}=h$ holds.

If $\pi\left(X^{\prime}\right) \simeq \mathbf{P}^{1}$, then $X^{\prime} \simeq X_{2}$ or $X^{\prime} \simeq \mathbf{P}^{1}$ since $\operatorname{deg}\left(\pi \circ \varphi_{X_{2}}\right)=2$. But $X^{\prime} \simeq \mathbf{P}^{1}$ cannot happen because no projective line can be contained in an abelian variety. Thus we have $X^{\prime} \simeq X_{2}$ and repeating the argument given above we complete the proof. 
Proof of Corollary 1.5. By the decomposition (1), any $\alpha \in H^{1}(X, \mathbf{C})$ can be written as $\alpha=\alpha_{1}+\overline{\alpha_{2}}$, where $\alpha_{1}, \alpha_{2} \in \mathcal{H}^{1}(X)$. Thus we can extend the homomorphism $\tau: \mathcal{H}^{1}\left(X_{2}\right) \rightarrow \mathcal{H}^{1}\left(X_{1}\right)$ to

$$
\widetilde{\tau}: H^{1}\left(X_{2}, \mathbf{C}\right) \rightarrow H^{1}\left(X_{1}, \mathbf{C}\right)
$$

by

$$
\widetilde{\tau} \alpha=\tau \alpha_{1}+\overline{\tau \alpha_{2}} .
$$

The extended homomorphism $\widetilde{\tau}$ also satisfies $f_{1}^{*} \circ \widetilde{\tau}=f_{2}^{*}: H^{1}\left(X_{2}, \mathbf{C}\right) \rightarrow H^{1}(\widetilde{X}, \mathbf{C})$, hence we can apply Theorem 1.4.

Proof of Corollary 1.6. Taking the tensor product with $\mathbf{C}$, we have a homomorphism of the first homology groups with $\mathbf{C}$-coefficients $H: H_{1}\left(X_{1}, \mathbf{C}\right) \rightarrow H_{1}\left(X_{2}, \mathbf{C}\right)$ which commutes with the induced homomorphisms $f_{i *}: H_{1}(\widetilde{X}, \mathbf{C}) \rightarrow H_{1}\left(X_{i}, \mathbf{C}\right)(i=$ $1,2)$, i.e., $f_{2 *}=H \circ f_{1 *}$. Considering the dual of the maps, we have

$$
{ }^{t} H: H^{1}\left(X_{2}, \mathbf{C}\right) \rightarrow H^{1}\left(X_{1}, \mathbf{C}\right)
$$

with

$$
f_{2}^{*}=f_{1}^{*} \circ{ }^{t} H: H^{1}\left(X_{2}, \mathbf{C}\right) \rightarrow H^{1}(\tilde{X}, \mathbf{C}) .
$$

Applying Theorem 1.4 to ${ }^{t} H$, we complete the proof.

Proof of Corollary 1.7. From the proof of Theorem 1.4, we see that the degree of $\left.n_{J(X)}\right|_{\varphi_{X}(X)}: \varphi_{X}(X) \rightarrow n_{J(X)} \circ \varphi_{X}(X)$ is one. Thus a pair of points $(p, q) \in X \times X$ with $n \cdot \varphi_{X}(p)=n \cdot \varphi_{X}(q)$ corresponds to a singular point of $n_{J(X)} \circ \varphi_{X}(X)$, and hence the number of such pairs $(p, q)$ is finite. Applying Abel's theorem to such pairs of points, we obtain the result.

\section{References}

[1] Accola, R. D. M.: Automorphisms of Riemann surfaces. - J. Analyse Math. 18, 1967, 1-5.

[2] FARKAS, H. M., and I. KRA: Riemann surfaces. - Springer-Verlag, New York-BerlinHeidelberg, 2nd edition, 1991.

[3] Gilman, J.: A matrix representation for automorphisms of compact Riemann surfaces. - Linear Algebra Appl. 17, 1977, 139-147.

[4] Hurwitz, A.: Ueber algebraische Gebilde mit eindeutigen Transformationen in sich. - Math. Ann. 41, 1892, 403-442.

[5] Lange, H., and Ch. Birkenhake: - Complex Abelian varieties. - Springer-Verlag, New YorkBerlin-Heidelberg, 1992.

[6] Martens, H. H.: Observations on morphisms of closed Riemann surfaces. - Bull. London Math. Soc. 10, 1978, 209-212.

[7] Martens, H. H.: Problems in the theory of closed Riemann surfaces. - Lecture Notes in Math. 747, 1979, 268-277.

[8] MirandA, R.: Algebraic curves and Riemann surfaces. - Amer. Math. Soc., Providence, RI, 1995.

[9] Springer, G.: Introduction to Riemann surfaces. - Addison-Wesley, Reading, Massachusetts, 1957.

[10] Tanabe, M.: Hurwitz' theorem and a generalization for holomorphic maps of closed Riemann surfaces. - Ann. Acad. Sci. Fenn. Math. 34, 2009, 391-399. 